\title{
An antibody to VacA of Helicobacter pylori in cerebrospinal fluid from patients with Guillain-Barre syndrome
}

\author{
S Chiba, T Sugiyama, K Yonekura, S Tanaka, H Matsumoto, N Fujii, S Ebisu, \\ K Sekiguchi
}

J Neurol Neurosurg Psychiatry 2002;73:76-78

Objective: To detect antibodies to recombinant vacuolating cytotoxin ( $\mathrm{r}$-VacA) of Helicobacter pylori in cerebrospinal fluid (CSF) from patients with Guillain-Barre syndrome (GBS).

Methods: CSF samples from 13 patients with GBS (electrophysiologically classified as eight acute inflammatory demyelinating polyradiculoneuropathy (AIDP), four acute motor axonal neuropathy (AMAN), and one unexcitable nerve conduction) and eight disease control patients were studied. The r-VacA protein was separated by SDS/ PAGE, and Western blot analysis was carried out.

Results: Six of the 13 patients with GBS had a specific lgG antibody to VacA of $H$ pylori, which was confirmed by absorption experiments using r-VacA. Every patient with positive CSF anti-r-VacA lgG had AIDP.

Conclusion: The sequence homology previously found between $\mathrm{VacA}$ and human $\left(\mathrm{Na}^{+}+\mathrm{K}^{+}\right)$-ATPase $\mathrm{A}$ subunit suggests that antibodies to $\mathrm{VacA}$ involve ion channels in abaxonal Schwann cell plasmalemma resulting in demyelination in some patients with GBS.

$\mathrm{R}^{\mathrm{s}}$ ecent neurophysiological studies on Guillain-Barre syndrome (GBS) have led to its reclassification as acute inflammatory demyelinating polyradiculoneuropathy (AIDP), acute motor axonal neuropathy (AMAN), and Fisher syndrome. ${ }^{1}$ Campylobacter jejuni is the most common antecedent pathogen of GBS. ${ }^{2}$ Many patients with GBS associated with $C$ jejuni infection contain serum antibodies to several gangliosides. However, a direct causal link to GBS has not been confirmed except for the correlation between GQlb antibody and Fisher syndrome.

Helicobacter pylori is a common cause of gastroduodenal disorders. Antigenic mimicry between $H$ pylori and the host is speculated to be a possible mechanism for the mucosal damage. ${ }^{3}$ We have reported the presence of several IgG antibodies against crude $\mathrm{H}$ pylori antigens in the cerebrospinal fluid (CSF) of patients with GBS. ${ }^{4}$ However, the nature of the antibody to the $90 \mathrm{kDa}$ protein has not been identified. In this study, we determined the $90 \mathrm{kDa}$ protein to be vacuolating cytotoxin (VacA) by Western blot analysis using recombinant (r)-VacA, and studied the clinical and electrophysiological features in patients with positive or negative antibody to $\mathrm{r}$-VacA in CSF.

\section{PATIENTS AND METHODS}

Thirteen patients with GBS ( 10 men and three women; mean age 52.1 years) were studied. They fulfilled the clinical criteria for GBS, ${ }^{5}$ and the electrophysiological studies were carried out within two weeks of onset.
Nerve conduction studies were performed on the ulnar and median nerves. The peroneal and sural nerves were tested when relevant. The electrodiagnostic variables used as criteria were: the compound muscle action potential; the distal motor latency; the motor nerve conduction velocity; the compound sensory nerve action potential the sensory nerve conduction velocity; and the F response latency.

Samples of CSF were obtained from 13 patients with GBS, in addition to four patients with definite multiple sclerosis (one man and three women; mean age 26.5 years) and four patients with motor neurone disease (one man and three women; mean age 61 years) as the disease controls. Informed consent was obtained from each patient and control subject.

The sense primer VacA-5 (ATGGATCCGCCTTTTTCACAACCGTGATC) containing a BamHI site and the anti-sense primer VacA-3 (ATCTGCAGTTAAGTGGTGTTGGTGGGTAA) containing PstI were synthesised. ${ }^{6}$ Chromosomal DNA was extracted from $H$ pylori NCTC 11637 with a SepaGene kit (Sanko Co, Tokyo, Japan) and used as a template for polymerase chain reaction. The polymerase chain reaction product was digested by BamHI and PstI and inserted at the downstream site of the EGF gene on the expression plasmid pNH400EGF-CYT3. The plasmid was transfected to the Bacillus brevis HPD31. The bacteria were grown for 48 hours at $30^{\circ} \mathrm{C}$ in $2 \mathrm{SL}$ medium, ${ }^{7}$ and then centrifuged and disrupted by ultrasonication (Branson Ultrasonics Co, Danbury, Connecticut, USA). The precipitate of the bacterial disruptant was solubilised with $20 \mathrm{mM}$ Tris/HCl ( $\mathrm{pH} 8.0$ ) containing $8 \mathrm{M}$ urea. The dissolved fraction was dialysed against $20 \mathrm{mM}$ Tris/HCl (pH 8.0)/l mM EDTA/l mM dithiothreitol/1 mM phenylmethanesulfonyl fluoride $/ 5 \%$ glycerol, and purified using a model 491 Prep-cell (Bio-Rad, Tokyo, Japan). The product was identified as a $93 \mathrm{kDa}$ fusion protein, consisting of $90 \mathrm{kDa}$-VacA and $3 \mathrm{kDa}$ EGF by Western blot analysis using an antibody to EGF.

Western blot analysis was carried out as reported. ${ }^{4}$ Briefly, 2 $\mu \mathrm{g}$ r-VacA was separated by SDS/PAGE using $12 \%$ acrylamide gel and transferred to a poly(vinylidene difluoride) microporous membrane (Millipore Corp, Tokyo, Japan). The membrane was incubated with a 120 -fold dilution of CSF for three hours and allowed to react with horseradish peroxidase labelled rabbit anti-human IgG at a 1:6000 dilution, and developed with an ECL developing solution (Amersham Pharmacia Biotech, Amersham, Bucks, UK). To detect the serum antibody to r-VacA, we also used a 200 -fold dilution of serum from three patients confirmed to have r-VacA antibody in their

Abbreviations: GBS, Guillain-Barre syndrome; AIDP, acute inflammatory demyelinating polyradiculoneuropathy; AMAN, acute motor axonal neuropathy; CSF, cerebrospinal fluid; VacA, vacuolating cytotoxin 


\begin{tabular}{|c|c|c|c|c|c|c|c|c|c|}
\hline $\begin{array}{l}\text { Patient } \\
\text { no }\end{array}$ & $\begin{array}{l}\text { Sex/ } \\
\text { Age }\end{array}$ & $\begin{array}{l}\text { Electrophysiological } \\
\text { classification }\end{array}$ & FNCV & $\begin{array}{l}\text { CSF } \\
\text { anti-r-VacA ab }\end{array}$ & $\begin{array}{l}\text { CSF IgG } \\
(\mathrm{mg} / \mathrm{dl})\end{array}$ & $\begin{array}{l}\text { Serum } \\
\text { anti-H.p. ab }\end{array}$ & $\begin{array}{l}\text { Serum } \\
\text { anti-r-VacA ab }\end{array}$ & $\begin{array}{l}\text { Brain stem } \\
\text { signs }\end{array}$ & Anti-ganglioside $a b$ \\
\hline 1 & $M / 78$ & AIDP & ND & Positive & 19.7 & 3.9 & NE & None & NE \\
\hline 2 & $M / 77$ & AIDP & NE & Positive & 8 & 4 & NE & None & Negative \\
\hline 3 & $M / 48$ & AIDP & WLatency & Positive & 8.8 & 5.5 & Positive & None & $\begin{array}{l}\text { GMlb lgG, M. } \\
\text { GalNAc GDla lgG, M }\end{array}$ \\
\hline 4 & $F / 54$ & AIDP & NE & Positive & 4.8 & 2.9 & NE & $\begin{array}{l}\text { Facial palsy } \\
\text { Abducens palsy }\end{array}$ & NE \\
\hline 5 & $M / 72$ & AIDP & $\downarrow$ Latency & Positive & 8.8 & 5.6 & Positive & None & Negative \\
\hline 6 & $F / 56$ & AIDP & Normal & Positive & 27.8 & NE & Positive & None & GM1 $\lg G$ \\
\hline 7 & $M / 56$ & Inexcitable & NE & Negative & 3.6 & 4 & NE & None & NE \\
\hline 8 & $M / 44$ & AMAN & Normal & Negative & 21.7 & NE & NE & None & NE \\
\hline 9 & $M / 48$ & AMAN & NE & Negative & 11.4 & $\begin{array}{l}309 \mathrm{U} / \mathrm{ml} \\
\text { (positive)* }\end{array}$ & NE & None & NE \\
\hline 10 & $M / 51$ & AIDP & Normal & Negative & 2.6 & 3 & NE & None & GMl $\lg G$ \\
\hline 11 & $M / 33$ & AMAN & Normal & Negative & 30.6 & Negative & NE & $\begin{array}{l}\text { Facial palsy } \\
\text { Bulbar signs }\end{array}$ & Negative \\
\hline 12 & $M / 40$ & AMAN & Normal & Negative & 12.3 & Negative & NE & None & Negative \\
\hline 13 & $F / 20$ & AIDP & Normal & Negative & 8.6 & Negative & NE & None & $\mathrm{NE}$ \\
\hline
\end{tabular}

CSF, cerebrospinal fluid; ab, antibody; H.p., Helicobacter pylori; NE, not examined; *: AMRAD Operation Pty LTD (Australia).

CSF. Serum antibody to $H$ pylori was measured with an electroimmunoassay kit using HM-CAP as antigen (Epi Co, Westbury, New York, USA).

\section{RESULTS}

According to the electrophysiological criteria, ${ }^{1}$ the patients were divided into three categories: eight patients with AIDP; four patients with AMAN; and one patient who showed unexcitable nerve conduction. Table 1 summarises their clinical backgrounds.

Six CSF samples from patients with GBS reacted with r-VacA protein in the Western blot (fig 1). The reaction disappeared when the samples had been mixed and absorbed by r-VacA for one hour at $37^{\circ} \mathrm{C}$. This indicates that these six patients had specific IgG antibodies to r-VacA protein in their CSF. No reaction was observed in the disease control patients.

The electrophysiological findings showed that every patient with positive CSF anti-r-VacA IgG had AIDP. The patients with negative anti-r-VacA IgG in the CSF were: two with AIDP, the four with AMAN, and the one with unexcitable nerve conduction. These results indicate that demyelination of the peripheral nerves was predominantly observed in patients with a positive CSF anti-r-VacA IgG. An F wave study, which indicates the involvement of proximal nerve conduction, was also carried out in nine patients. Although serum from only three of the six patients with positive CSF anti-r-VacA IgG were examined, two of these three had delayed $F$ wave latencies.

With respect to the correlation between serum antibody to crude $H$ pylori antigens and the CSF anti-r-VacA IgG, five of the six patients with a positive CSF anti-r-VacA IgG had a positive serum antibody. In contrast, three of the six patients with a negative CSF anti-r-VacA IgG displayed positive serum antibody. There was no significant difference in CSF IgG concentrations between patients who tested positive (13.2 (8.4) $\mathrm{mg} / \mathrm{dl}$; mean (SD)) and negative (13.0 (9.3) $\mathrm{mg} / \mathrm{dl})$ for antibody to r-VacA.

\section{DISCUSSION}

We found that six of 13 CSF samples from patients with GBS had specific IgG antibodies to r-VacA, and the patients with anti-r-VacA IgG showed appreciable demyelination of the peripheral nerves.

Considering the cause of GBS, $C$ jejuni induces immune responses through serum anti-ganglioside antibodies because of shared homologous epitopes, and cross reacts with the surface components of peripheral nerves. ${ }^{8}$ Immune reactions to epitopes in the Schwann cell, or myelin, may be the result in some patients with AIDP. Reactions against the axonal membrane are thought to be involved in the development of AMAN. ${ }^{9}$ However, recent studies showed that anti-ganglioside antibody was commonly detected in the serum of patients with AMAN and not in serum of patients with AIDP. ${ }^{10}{ }^{11}$ Consequently, the exact mechanism of AIDP remains unsolved. In addition, the CSF of patients with GBS has not been fully investigated. An attractive hypothesis for the pathophysiology of AIDP is that an unknown antibody to antigens on the outer surface of the abaxonal Schwann cell plasmalemma binds complement, resulting in the development of transmembrane pores. $^{11}$

Although $H$ pylori infection is one of the major causes of gastroduodenal diseases, the antibody to $H$ pylori reacts with some extragastric tissues, ${ }^{12}$ and some autoimmune diseases regress after eradication of $H$ pylori. ${ }^{13}$ VacA has been reported to form anion selective, low conductance, voltage dependent channels in planar membranes. ${ }^{14}$ The formation of low conductance VacA pores in the cell membrane results in a change in ion permeability. ${ }^{15}$ In addition, sequence homology between VacA and the human $\left(\mathrm{Na}^{+}+\mathrm{K}^{+}\right)$-ATPase A subunit has been reported. ${ }^{16}$ Concerning the association between serum and CSF antibodies, every patient with positive CSF anti-r-VacA IgG had positive serum antibodies to $H$ pylori, and two patients with serum anti-r-VacA IgG showed delayed F wave latencies.

The peripheral nervous system is protected by the blood/nerve barrier, which is more vulnerable than the central

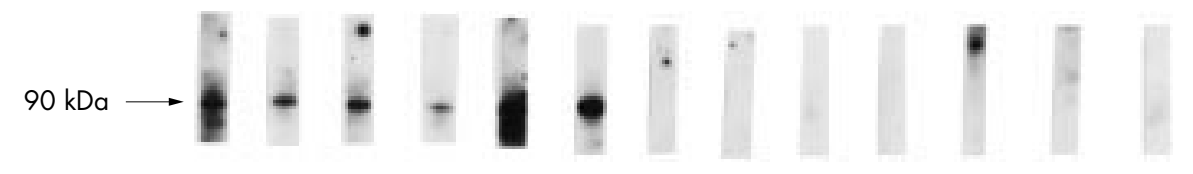

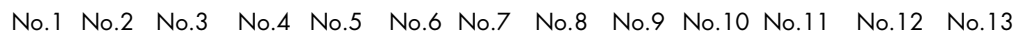

Figure 1 The reactivity of cerebrospinal fluid antibodies to recombinant VacA in patients with Guillain-Barre syndrome (GBS). The main band, compatible with the antibody to the $90 \mathrm{kDa}$ protein, was seen in six of the samples of cerebrospinal fluid from 13 patients with GBS. 
nervous system to antibody mediated disorders because it is not as tight as the blood/brain barrier. ${ }^{11}$ However, the present data suggest that local immune responses may be easily facilitated in the intraendoneurial space of the peripheral nervous system, because the profile of CSF antibody to $H$ pylori antigens was quite different from that of serum. ${ }^{4}$ In addition, the patients with positive anti-r-VacA IgG in CSF showed diffuse demyelination of the peripheral nerves. Our findings suggest that anti-VacA IgG in the CSF not only changes the permeability of the cell membrane through ion channel dysfunction, but may also involve the abaxonal Schwann cell plasmalemma in some patients with GBS. However, the exact mechanism of the production of anti-VacA IgG in CSF remains unknown.

\section{ACKNOWLEDGEMENTS}

We acknowledge the encouragement and advice from Dr Akira Yachi (former President of Sapporo Medical University, Sapporo, Japan).

\section{Authors' affiliations}

S Chiba, K Yonekura, S Tanaka, H Matsumoto, Department of Neurology, School of Medicine, Sapporo Medical University, Sapporo, Japan

N Fujii, Department of Microbiology, School of Medicine, Sapporo Medical University

T Sugiyama, Department of Gastroenterology, Hokkaido University Graduate School of Medicine, Sapporo

S Ebisu, Higeta Shoyu Co Ltd, Chiba, Japan

K Sekiguchi, Institute of Immunology, Tochigi, Japan

Correspondence to: Dr Chiba, Department of Neurology, School of Medicine, Sapporo Medical University, Minami 1-Jo Nishi 16 chome, Chuo-ku, Sapporo 060-8543, Japan; chiba@sapmed.ac.jp

Received 4 September 2001

In revised form 24 January 2002

Accepted 27 February 2002

\section{REFERENCES}

1 Ho TW, Li CY, Cornblath DR, et al. Patterns of recovery in the Guillain-Barre syndrome. Neurology 1997;48:695-700.

2 Rees JH, Soudain SE, Gregson NA, et al. Campylobacter jejuni infection and Guillain-Barre syndrome. N Engl J Med 1995;333:1374-9.

3 Negrini R, Savio A, Poiesi C, et al. Antigenic mimicry between $\mathrm{H}$. pylori and gastric mucosa in the pathogenesis of body atrophic gastritis. Gastroenterology 1996;111:655-65.

4 Chiba S, Sugiyama T, Matsumoto H, et al. Antibodies against Helicobacter pylori were detected in the cerebrospinal fluid obtained from patients with Guillain-Barre syndrome. Ann Neurol 1998:44:686-8.

5 Asbury AK, Cornblath DR. Assessment of current diagnostic criteria for Guillain-Barre syndrome. Ann Neurol 1990;27(suppl):S21-4.

6 Schmitt W, Haas R. Genetic analysis of Helicobacter pylori vacuolating cytotoxin: structural similarities with the lgA protease type of exported protein. Mol Microbiol 1994;12:307-19.

7 Miyauchi A, Ebisu S, Uchida K, et al. Pilot scale production of a recombinant human epidermal growth factor, secreted by Bacillus brevis, using expanded absorption. Journal of Industrial Microbiology and Biotechnology 1998;21:208-14

8 Yuki N, Handa S, Taki T, et al. Cross-reactive antigen between nervous tissue and a bacterium elicits Guillain-Barre syndrome: molecular mimicry between ganglioside GMI and lipopolysaccharide from Penner's serotype 19 of Campylobacter jenuni. Biomedical Research 1992;13:451-3.

9 Hahn AF. Guillain-Barre syndrome. Lancet 1998;352:635-41.

10 Ogawara K, Kuwabara S, Mori M, et al. Axonal Guillain-Barre syndrome: relation to anti-ganglioside antibodies and Campylobacter jenuni infection in Japan. Ann Neurol 2000;48:624-31.

11 Ho TW, McKhann GM, Griffin JW. Human autoimmune neuropathies. Annu Rev Neurosci 1998;21:187-226.

12 Ko GH, Park HB, Shin MK, et al. Monoclonal antibodies against Helicobacter pylori cross-react with human tissue. Helicobacter 1997;2:210-15

13 Gasbarrini A, Franceschi F, Tartaglione R, et al. Regression of autoimmune thrombocytopenia after eradication of Helicobacter pylori. Lancet 1998;352:878.

14 Tombola F, Carlesso C, Szabo I, et al. Helicobacter pylori vacuolating toxin forms anion-selective channels in planar lipid bilayers: possible implications for the mechanism of cellular vacuolation. Biophys J 1999;76:1401-9.

15 Szab I, Brutsche S, Tombola F, et al. Formation of anion-selective channels in the cell plasma membrane by the toxin VacA of Helicobacter pylori is required for its biological activity. EMBO J 1999:18:5517-27.

16 Cover TL, Blaser M. Purification and characterization of the vacuolating toxin from Helicobacter pylori. J Biol Chem 1992;267:10570-5. 